\title{
Future perspective of induced pluripotent stem cells for diagnosis, drug screening and treatment of human diseases
}

\author{
Qizhou Liann ${ }^{1,2}$; Yenyen Chow ${ }^{1,2}$; Miguel Angel Esteban ${ }^{3}$; Duanqing Pei ${ }^{3}$; Hung-Fat Tse ${ }^{1}$ \\ ${ }^{1}$ Cardiology Division, Department of Medicine, University of Hong Kong, Hong Kong, China; ${ }^{2}$ Research Centre of Heart, Brain, Hormone, and Healthy Aging, University of Hong \\ Kong, Hong Kong, Hong Kong, China; ${ }^{3}$ Stem Cell and Cancer Biology Group, Key Laboratory of Regenerative Biology, South China Institute for Stem Cell Biology and Regenerative \\ Medicine, Guangzhou Institutes of Biomedicine and Health, Chinese Academy of Sciences, Guangzhou, China
}

\begin{abstract}
Summary
Recent advances in stem cell biology have transformed the understanding of cell physiology and developmental biology such that it can now play a more prominent role in the clinical application of stem cell and regenerative medicine. Success in the generation of human induced pluripotent stem cells (iPS) as well as related emerging technology on the iPS platform provide great promise in the development of regenerative medicine. Human iPS cells show almost identical properties to human embryonic stem cells (ESC) in pluripotency, but avoid many of their limitations of use. In addition, investigations into reprogramming
\end{abstract}

Correspondence to:

Hung-Fat Tse, MD, PhD

Cardiology Division, Department of Medicine

Li Ka Shing Faculty of Medicine

The University of Hong Kong

Rm 1928, Block K, Queen Mary Hospital

Hong Kong, HKSAR, China

Tel.: +852 2553598, Fax: +852 28186304

E-mail:hftse@hkucc.hku.hk of somatic cells to pluripotent stem cells facilitate a deeper understanding of human stem cell biology. The iPS cell technology has offered a unique platform for studying the pathogenesis of human disease, pharmacological and toxicological testing, and cell-based therapy. Nevertheless, significant challenges remain to be overcome before the promise of human iPS cell technology can be realised.

\section{Keywords}

Human induced pluripotent stem cells, reprogramming, in vitro disease models, drug screening

Received: May 13, 2010

Accepted after minor revision: May 26, 2010

Prepublished online: June 10, 2010

doi:10.1160/TH10-05-0269

Thromb Haemost 2010; 104: 39-44

\section{Introduction}

The initial aim of stem cell biology is to provide insight into how genetic information is related to tissue and organ formation. Recent advances in this field nonetheless also show great promise in the development of regenerative medicine since many types of pluripotent stem cell can give rise to differentiated progeny in vitro and tissue formation in the human body. In addition, stem cell platforms that employ cell lineages derived from human stem cells represent a novel approach for drug development and screening (1). Human embryonic stem cells (ESC) derived from early blastocysts are the prototype of pluripotent stem cells that are capable of unlimited growth in tissue culture and can differentiate into all cell types in the body. As a result, human ESCs lines have been generated and used to study the relationships between gene function and tissue formation and organogenesis (2). Human ESC-derived specific cell types have also been explored for cell-based therapies for tissue regeneration as well as cell-based assays for drug screening (3).

The development of a human ESC platform is limited by the low efficacy in establishing human ESC lines, especially patientspecific ESCs via somatic cell nuclear transfer, the moral and ethi- cal issues related to the use of human oocytes, as well as destruction of human blastocysts, and the immune rejection with allogeneic transplantation. One of the major recent breakthroughs in the field of stem cell biology has been the reprogramming of somatic cells into pluripotent cells by ectopic expression of transcription factors. In 2006, Takahashi and Yamanaka first reported that forced expression of four transcription factors: octamer 3/4 (Oct4), SRY box-containing gene 2 (Sox-2), Kruppel-like factor 4 (Klf4) and c-Myc out of an initial 24 factors screened, could reprogram mouse somatic fibroblasts into ESC-like colonies. These were termed induced pluripotent stem (iPS) cells (4). In 2007, Takahashi et al. and two other groups announced almost simlutaneously their success in human iPS cell generation (5-7). Further enhancement of the technique, such as the use of Nanog and Oct4, rather than F-box protein (Fbx15) as selection have made human iPS cells to display almost identical properties to ESCs in terms of multi-lineage invitro differentiation, teratoma formation, germline transmission, and even contribution to entire animals $(8,9)$. Nevertheless, there remain differences in gene expression patterns between iPS and ESCs (10). The generation of human iPS cells has circumvented the limitations of using human ESCs as discussed above. Thus iPS cell technology has opened up new avenues in biomedical research. In 
this review, the potential application of a patient-specific iPS cells as a platform for disease modelling and drug screening, as well as cell and tissue replacement therapies will be discussed ( Fig. 1).

\section{Recent advances in techniques to create iPS cells}

The details of different methodologies to create iPS cells are beyond the scope of this review (11), thus it will confine itself to recent important advances. In addition to the initial use of fibroblasts for reprogramming, the use of various other somatic cell types, including melanocytes, mesenchymal cells, peripheral blood cells, and adipose stem cells, has been described (12). The different cell types used for reprogramming might lead to in variation of efficacy of reprogramming and differentiation potential. Among the different protocols used for reprogramming, Oct4 is believed to be the essential component and the other transcription factors can be compensated by the endogenous expression by initial cell types (12) or exogenous supplement of small molecules, such as DNA methyltransferase inhibitors and the histone deacetylase inhibitor valproic acid $(12,13)$. The use of genome-integrating viral vectors such as retroviruses and lentiviruses for reprogramming is associated with altered gene expression (14), and a potential risk of reactivation of viral transgenes. As a result, such iPS cells are inappropriate for therapeutic use: they may affect the phenotypes of their derived cells for disease modelling. Nevertheless, recent studies reveal that genome-integrating viral vectors can be removed using the Cre-lox system $(13,14)$. Other techniques that do not require genome-integrating viral vectors, such as adenoviral, episomal and sendai virus vectors, repeated plasmid transfection, arginine peptide tagged proteins and small molecules, have also been reported (12-14).

\section{Disease modelling}

Animal models can provide useful information about disease mechanisms, but they are limited by their fundamental differences in genetic background, physiology and pathophysiology compared with humans. Nonetheless it is unreasonable to use cell biology techniques for the investigation of human disease mechanisms using human tissue as in general this requires large amounts of live human cells and tissues from affected patients. Thus, the use of human stem cell platforms to derive different cells and tissues in the human body for disease modelling is an attractive option.

The promise of using ESCs, and more recently iPS cells, to model human disease is based on the unique capacity of these cells to continuously self-renew and differentiate into all cell types in the body. By capturing the entire genetic repertoire of an individual with a disease, one may be able to duplicate the pathogenesis of that individual's disease 'in a dish'. In order to apply a human ESC platform to disease modelling, over-expression of known diseasecausing genes or a derived ESC line from pre-implantation embryos with a known genetic mutation are needed. This method is thus confined to diseases with a documented genetic defect.

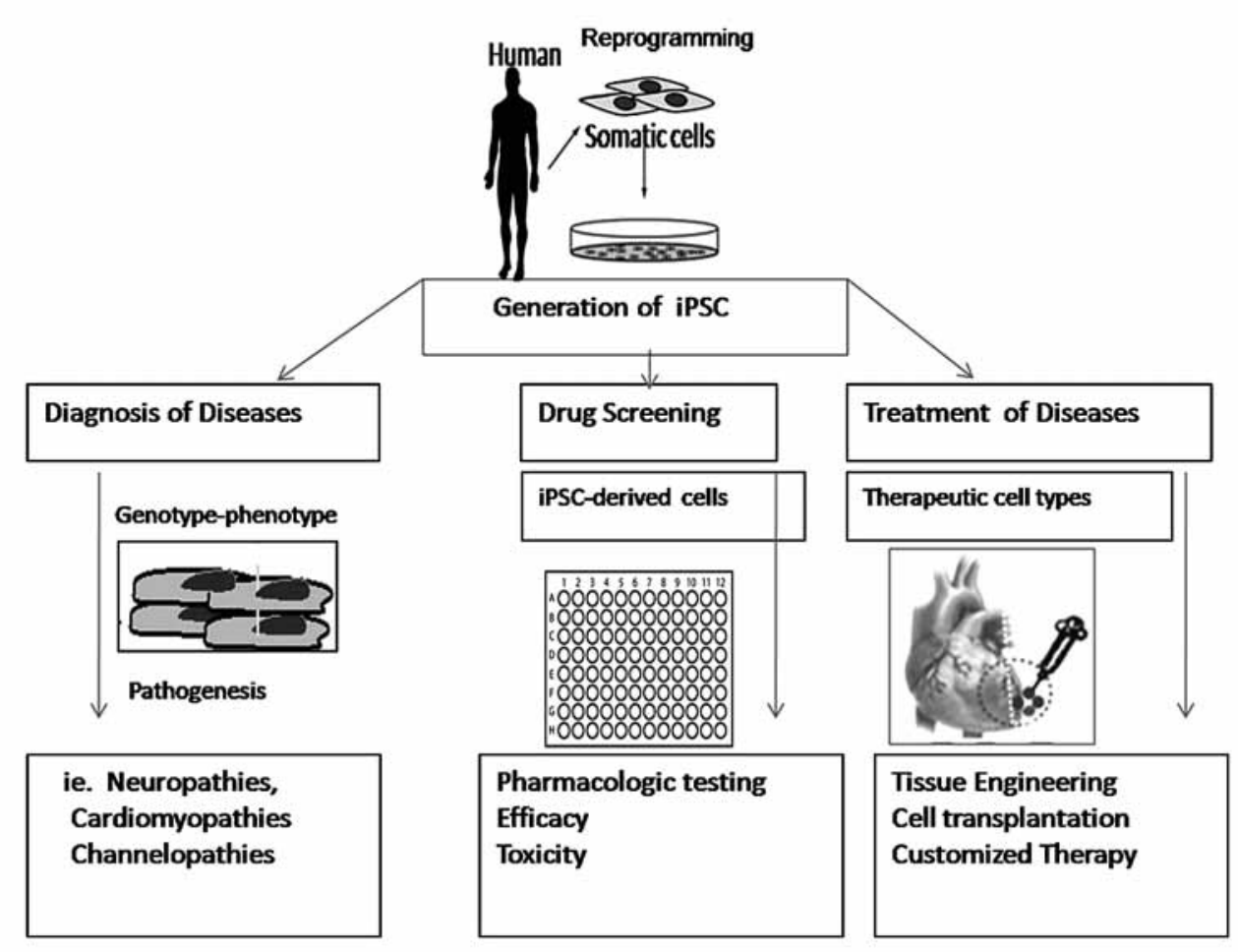

Figure 1: Potential clinical application of induced pluripotent stem cells for diagnosis, drug screening and treatment of human diseases. 
The overwhelming advantage of iPS cell technology over ES cells is that patient-specific iPS cells are easily generated $(15,16)$. This is an important benefit given that many genetic diseases are of a sporadic nature with no family history. More than 10 human disease-specific iPS cell lines have been established, ranging from simple single gene deficiencies to complex multifactorial diseases of unknown genetic origin, such as type 1 diabetes $(12,13,15)$. In addition, iPS technology will offer the unprecedented possibility of using human cells to study human diseases for which there are no animal models, such as Brugada syndrome and hypertrophic cardiomyopathy.

Recent studies have shown that patient-specific iPS cells can be used for human disease modelling. In 2009, Ebert et al. (17) reported the successful generation of iPS cells from a child with a genetic form of spinal muscular atrophy. These cells maintained the disease genotype, and were able to differentiate into motor neurons that showed selective deficits in survival of motor neuron protein aggregates, and were similar to the phenotype associated with motor neuron disease. Subsequently, Lee et al. have successfully generated patient-specific iPS cells from patients with familial dysautonomia, a neuropathy caused by a point mutation in the IKB kinase complex-associated protein (IKBKAP) gene (18). In addition to demonstrating the tissue-specific splicing defect in such iPS cell-derived tissue, the specific defects of the nervous systems were also duplicated using this technique. In both studies, the patientspecific iPS cell platform was used to provide new insight into the disease itself as well as potential new targeted treatment. Agarwal et al. (19) demonstrated that patient-specific iPS cells with dyskeratosis congenita could provide novel insight into the disease pathology. Patients with dyskeratosis congenita suffer from degeneration in multiple tissues due to disordered telomere maintenance. Reprogramming human somatic cells in these patients leads to telomere elongation and consequent correction of the defective telomerase RNA component in this disorder. In addition, these findings suggest that strategies to reverse this defective telomerase RNA component may offer a potential new therapeutic approach for patients with dyskeratosis congenita.

There remain nonetheless several major challenges to the use of iPS technology in modelling more complex diseases $(12,13,15)$. First, late-onset human diseases have a long latency before the phenotypes can be manifested in the culture system. Therefore different approaches, such as exposure to oxidative stress and hypoxia in the cell culture system, have been proposed to accelerate the pathological phenotypes and reveal the potential susceptibility to these environmental stresses. Second, some of the disease processes may not be manifested by a single purified lineage-committed cell type. As a result, more than one cell type may be required during the coculture assay. Third, diseases with a potential epigenetic contribution, such as those caused by combined genetic and environmental factors, may be difficult to study since the reprogramming process during generation of iPS cells should, in principle, remove any epigenetic changes. Overall though, iPS technology expands the horizon for studying pathogenesis in a culture dish. It will not completely replace current tools, such as transgenic animal models to study more complex diseases as well as their in vivo aspect.

\section{Drug screening}

Current drug discovery and development programs are inefficient. Currently, more than $90 \%$ of lead candidates identified by the current in vitro screening systems fail to become drugs due to safety and efficacy issues in clinical applications. Although genetically modified rodents, immortalised human cell lines and animal models have provide useful information in studying safety and efficacy of drugs, their major weakness is that they fail to replicate human conditions. The treatment response to drugs in animal models cannot be used to predict efficacy in humans. For example, creatine (20) is very effective in altering the disease characteristics of amyotrophic lateral sclerosis due to over-expression of mutant superoxide dismutase in a transgenic mouse model. Nonetheless, no clinical improvements have been observed in human clinical trials (21). Individual variability in response to potential therapeutic agents also cannot be tested using the current drug testing platform $(12,22)$. Additional drug screening model systems are thus needed to better mimic human conditions (16).

The iPS cell technology may provide a novel approach to pharmacological and toxicological testing $(12,13,16)$. First, the iPS cell platform allows the generation of human disease-specific cell types to enable better prediction of therapeutic response and toxicology, for example neurons, cardiomyocytes, and hepatocytes. Second, a library of different iPS cell lines for the same human disease can be generated and provide insight into the genetic and potentially epigenetic variation of a broad section of the population. Third, the variation in therapeutic effect of a potential drug can be tested at an individual level. Personalised medicine thus becomes a reality with the use of patient-specific iPS cells.

Cardiac and liver toxicity are a major cause of drug failure during pre-clinical and clinical testing. The lack of an in vitro model to detect pro-arrhythmic effects of drugs on human heart cells has hindered the development of many therapeutic agents: iPS cell-derived cardiomyocytes $(23,24)$ can provide a valuable cell source to test drug efficacy and safety prior to clinical testing. The use of iPS cell-derived cardiomyocytes from patients with long-QT syndrome may be used as a model to test the safety of potential candidate agents against this lethal arrhythmia. Treatment response of many genetic disorders known to affect heart function, such as dilated cardiomyopathy, can also be determined with this iPS cell platform. Recent studies have validated this concept using human iPS cell-derived cardiomyocytes together with a multi-electrode assay as a platform to study the changes in electrophysiological properties of heart cells with different agents $(25,26)$.

Disease- or patient-specific iPS cells thus have great potential in pharmaceutical development. The iPS cell technology can be applied in high-throughput screening assays and also used in prediction of individual patient response to therapeutic agents. In the early-stages of drug development, the use of iPS cell technology can substantially minimise the number of animals sacrificed during drug testing, enable early human toxicity to be detected in preclinical trials, and decrease the risk and cost associated with clinical trials. While iPS technology provides great opportunities for drug screening and toxicity testing, we should be aware that cells 
generated from iPS cells are developmentally immature, and may better represent a model for fetal biology. In order to reflect adult human biology, maturation of the iPS-derived cells is required prior to their use for drug development. The provision of lineage specific reporter lines through genetic manipulation to drive the differentiation and maturation of iPS cells to specific cell types should enhance this development.

\section{Cell-based therapy}

Following the success of hematopoietic stem cell therapy in the treatment of haematological diseases, the potential application of cell based therapy has been extended to the treatment of other human diseases. In particular, different types of adult stem cells, including bone marrow, peripheral haematopoietic, and mesenchymal stem cells (MSC) have been evaluated in the treatment of cardiovascular disease (Table 1) (27-29). Nonetheless adult stem cells have limited potential for proliferation and differentiation, thus ESCs have been explored for tissue regeneration as they can be differentiated into various therapeutic relevant cell types in vitro (30). Despite this, there is limited progress in the use of ESCs for tissue regeneration in humans due to various technical, social and religious issues (12).

The generation of patient-specific iPS cells has the advantage of avoiding many of the ethical concerns associated with the use of embryonic or fetal material, and have no risk of immune rejection. Currently, several therapeutic relevant cell types, including motor neuron (31), hepatocytes $(32,33)$, pancreatic insulin producing cells (34), haematopoietic cells (35-37), retinal cells (38), cardiomyocyte $(39,40)$ and MSCs $(41)$, have been successfully derived from human iPS cells, and some of them have been tested to treat diseases in animal models. In murine models of haemophilia $A$ and sickle cell anaemia, iPS cell-based therapies have been shown to attenuate the disease process $(35,36)$. The use of iPS cells has thus been proposed as diagnostic and therapeutic tools for different haematological disorders (37). Carr et al. (38) have demonstrated that transplantation of human iPS cell-derived retinal cells can rescue retinal dysfunction in rats. This suggests that retinal cells derived from healthy iPS cells are functional, and can potentially be used to treat retinal dysfunction. For cardiovascular regeneration, different iPS cell-derived cell types, including cardiomyocytes (23, 40) or MSCs (41) have been successfully generated. Nelson et al. (39) first reported the use of iPS cells for myocardial repair in animal models of acute myocardial infarction. Lian et al. (41) demon-

\begin{tabular}{|c|c|c|}
\hline Cell types & Advantage & Disadvantage \\
\hline Embryonic stem cells & $\begin{array}{l}\text { Pluripotent and unlimited supply } \\
\text { Patient-specific cells for autologous transplan- } \\
\text { tation possible via therapeutic cloning }\end{array}$ & $\begin{array}{l}\text { - Social and ethical concerns } \\
\text { - Risk of rejection and required immunosuppression } \\
\text { for allogenic transplant } \\
\text { - Limited supply of human oocytes } \\
\text { - Risk of tumor formation } \\
\text { - Proarrhythmic risk due to immature phenotype of } \\
\text { derived cardiomyocyte }\end{array}$ \\
\hline $\begin{array}{l}\text { Induced pluripotent } \\
\text { stem cells }\end{array}$ & $\begin{array}{l}\text { Pluripotent and unlimited supply } \\
\text { Patient-specific cells for autologous transplan- } \\
\text { tation possible }\end{array}$ & $\begin{array}{l}\text { - Risk of tumor formation } \\
\text { - Risk of viral vector } \\
\text { Proarrhythmic risk due to immature phenotype of } \\
\text { derived cardiomyocyte }\end{array}$ \\
\hline Skeletal myoblast & $\begin{array}{l}\text { - Autologous transplantation without the need } \\
\text { for immunosuppression or risk of rejection } \\
\text { - Can be expanded in vitro with high yield, } \\
\text { resistant to ischemia \& fatigue }\end{array}$ & $\begin{array}{l}\text { - Cannot differentiate into cardiomyocyte phenotype } \\
\text { - Lack of integration with host cardiomyocyte with } \\
\text { arrhythmogenic potential }\end{array}$ \\
\hline $\begin{array}{l}\text { Bone marrow } \\
\text { stem cells }\end{array}$ & $\begin{array}{l}\text { Autologous transplantation without the need } \\
\text { for immunosuppression or risk of rejection } \\
\text { - Can induce angiogenesis, possible pluripotent }\end{array}$ & $\begin{array}{l}\text { - Limited ability to differentiate into cardiomyocyte } \\
\text { - Limited supply and need for in-vitro expansion } \\
\text { - Difficult to isolate and propagate in culture }\end{array}$ \\
\hline $\begin{array}{l}\text { Mesenchymal } \\
\text { stem cells }\end{array}$ & $\begin{array}{l}\text { Autologous transplantation without the need } \\
\text { for immunosuppression or risk of rejection } \\
\text { - Can induce angiogenesis and possible pluripo- } \\
\text { tent } \\
\text { Lower risk of rejection and ? possible for } \\
\text { allogenic transplantation }\end{array}$ & $\begin{array}{l}\text { - Limited ability to differentiate into cardiomyocyte } \\
\text { - Limited supply and need for in-vitro expansion } \\
\text { - Difficult to isolate and propagate in culture }\end{array}$ \\
\hline Adult cardiac stem cells & $\begin{array}{l}\text { - Cardiomyocyte phenotype with no need for } \\
\text { differentiation } \\
\text { - Can integrate with host cardiomyocyte } \\
\text { - Autologous transplantation without the need } \\
\text { for immunosuppression or risk of rejection }\end{array}$ & $\begin{array}{l}\text { - Very limited supply } \\
\text { - Difficult to isolate and propagate in culture } \\
\text { - Proarrhythmic risk due to immature phenotype of } \\
\text { derived cardiomyocyte }\end{array}$ \\
\hline
\end{tabular}

Table 1: Different types of stem cells for cardiovascular diseases. 
strated that human iPS cell-derived MSCs are superior to adult bone marrow-derived MSCs in the attenuation of hindlimb ischaemia in mice. Despite the similarities between phenotypes of MSCs derived from bone marrow and iPS cells, those derived from iPS cells had better survival and engraftment following transplantation to induce vascular and muscle regeneration via direct de novo vascular and muscle differentiation and paracrine mechanisms. Therefore, stem cells derived from pluripotent stem cells seem to have better therapeutic efficacy compared with those from adult stem cell sources.

There are several major challenges to overcome before iPS cell technology is applied in clinical practice. First, current iPS cells are not "clinical grade". Genome-integrating viral vectors used for reprogramming are known oncogenes, particularly c-Myc, Oct4 and Klf4, such that iPS cells thus generated are unlikely to be safe for clinical application. Nonetheless recent technological advances, including reprogramming without viral integration such as plasmids or direct reprogramming protein delivery assays can solve this issue $(12,13)$. Second, the efficiency of human iPS cell generation using classic retroviral-mediated reprogramming is as low as $0.001 \%-0.01 \%$. Further improvement in iPS technologies, such as the inhibition of p53-mediated pathways $(42,43)$ and vitamin C supplementation (44), are needed to enhance the generation of iPS cells. Third, it remains unclear whether the human iPS cell clone has complete nuclear reprogramming. The stringent pluripotency state, as verified by the tetraploid complementation assay in mice (9), is not applicable to humans. Incomplete reprogramming of somatic cells to iPS cells could result in impaired differentiation of iPS cells into the required cell type (45). Finally, all pluripotent stem cells have the potential for teratoma formation. Stringent tests are thus required to ensure that all iPS cell-derived therapeutic cell types do not contain any undifferentiated iPS cells prior to transplantation. In the mouse model, teratoma formation can be seen with as few as 10,000 undifferentiated human ES cells (46).

In future, novel technologies must be developed to track cell fate in vivo for pre-clinical and clinical trials. Despite the challenges in the therapeutic use of iPS cells, preclinical studies have provided the proof-of-concept that patient-specific iPS cells can provide an unlimited cell source to produce massive therapeutic cell types, such as cardiomyocytes and MSCs, and can be prepared in an "offthe-shelf" format for cell transplantation.

\section{Future perspectives}

A decade of studies in human ESCs has yielded remarkable progress and understanding in stem cell biology. The technical challenge of creating patient-specific ESCs, the ethical issues arising from the fetal origin of human ESCs and the potential risk of immune rejection make broad clinical application of this cell type difficult. Recent advances in human iPS cell technology can potentially circumvent these disadvantages: iPS cells thus provide an invaluable resource of cell types for modelling diseases, drug or toxicology screening, and patient-specific cell therapy. Significant challenges remain to be overcome before the full potential of human iPS cell technology can be realised.

\section{Acknowledgements}

This study was supported by Collaborative Research Fund of Hong Kong Research Grant Council (HKU 8/CRF/09), NSFC/RGC Joint Research Scheme (N_HKU 716/09 to Q Lian ), and Small Project Funding (200907176179 to Q Lian) and Strategic Research Theme on Healthy Ageing (Q Lian and HF Tse) from University of Hong Kong.

\section{References}

1. Thomson JA, Itskovitz-Eldor J, Shapiro SS, et al. Embryonic stem cell lines derived from human blastocysts. Science 1998; 282: 1145-1147.

2. Rideout WM 3rd, Hochedlinger K, Kyba M, et al. Correction of a genetic defect by nuclear transplantation and combined cell and gene therapy. Cell 2002; 109: $17-27$.

3. Siu CW, Moore JC, Li RA. Human embryonic stem cell-derived cardiomyocytes for heart therapies. Cardiovasc Hematol Disord Drug Targets 2007; 7: 145-152.

4. Takahashi K, Yamanaka S. Induction of pluripotent stem cells from mouse embryonic and adult fibroblast cultures by defined factors. Cell 2006; 126: 663-676.

5. Takahashi K, Tanabe K, Ohnuki M, et al. Induction of pluripotent stem cells from adult human fibroblasts by defined factors. Cell 2007; 131: 861-872.

6. Park IH, Zhao R, West JA, et al. Reprogramming of human somatic cells to pluripotency with defined factors. Nature 2008; 451: 141-146.

7. Yu J, Vodyanik MA, Smuga-Otto K, et al. Induced pluripotent stem cell lines derived from human somatic cells. Science 2007; 318: 1917-1920.

8. Maherali N, Sridharan R, Xie W, et al. Directly reprogrammed fibroblasts show global epigenetic remodeling and widespread tissue contribution. Cell Stem Cell 2007; 2: 55-70.

9. Zhao XY, Li W, Lv Z, et al. iPS cells produce viable mice through tetraploid complementation. Nature 2009; 461: 86-90.

10. Chin MH, Mason MJ, Xie W, et al. Induced pluripotent stem cells and embryonic stem cells are distinguished by gene expression signatures. Cell Stem Cell 2009; 5: 111-123.

11. Maherali N, Hochedlinger K. Guidelines and techniques for the generation of induced pluripotent stem cells. Cell Stem Cell 2008; 3: 595-605.

12. Kiskinis E, Eggan K. Progress toward the clinical application of patient-specific pluripotent stem cells. J Clin Invest 2010; 120: 51-59.

13. Saha K, Jaenisch R. Technical challenges in using human induced pluripotent stem cells to model disease. Cell Stem Cell 2009; 5: 584-595.

14. Soldner F, Hockemeyer D, Beard C, et al. Parkinson's disease patient-derived induced pluripotent stem cells free of viral reprogramming factors. Cell 2009; 136: 964-977.

15. Colman A, Dreesen O. Pluripotent stem cells and disease modeling. Cell Stem Cell 2009; 5: 244-247.

16. Ebert AD, Svendsen CN. Human stem cells and drug screening: opportunities and challenges. Nat Rev Drug Discov 2010; 9: 367-372.

17. Ebert AD, Yu J, Rose FF Jr, et al. Induced pluripotent stem cells from a spinal muscular atrophy patient. Nature 2009; 457: 277-280.

18. Lee G, Papapetrou EP, Kim H, et al. Modelling pathogenesis and treatment of familial dysautonomia using patient-specific iPSCs. Nature 2009; 461: 402-406.

19. Agarwal S, Loh YH, McLoughlin EM, et al. Telomere elongation in induced pluripotent stem cells from dyskeratosis congenita patients. Nature 2010; 464: 292-296.

20. Klivenyi P, Ferrante RJ, Matthews RT, et al. Neuroprotective effects of creatine in a transgenic animal model of amyotrophic lateral sclerosis. Nat Med 1999; 5: 347-350.

21. Shefner JM, Cudkowicz ME, Schoenfeld D, et al. NEALS Consortium. A clinical trial of creatine in ALS. Neurology 2004; 631656-631661.

22. Rubin LL. Stem cells and drug discovery: the beginning of a new era? Cell 2008; 132: 549-552.

23. Zhang J, Wilson GF, Soerens AG, et al. Functional cardiomyocytes derived from human induced pluripotent stem cells. Circ Res 2009; 104: e30-41. 
24. Shiba Y, Hauch KD, Laflamme MA. Cardiac applications for human pluripotent stem cells. Curr Pharm Des 2009; 15: 2791-2806.

25. Tanaka T, Tohyama S, Murata $M$, et al. In vitro pharmacologic testing using human induced pluripotent stem cell-derived cardiomyocytes. Biochem Biophys Res Commun 2009; 385: 497-502.

26. Yokoo N, Baba S, Kaichi S, et al. The effects of cardioactive drugs on cardiomyocytes derived from human induced pluripotent stem cells. Biochem Biophys Res Commun 2009; 387: 482-488.

27. Siu CW, Liao SY, Liu Y, et al. Stem cells for myocardial repair. Thromb Haemost 2010; 104: 6-12.

28. Lawall H, Bramlage P, Amann B. Stem cell and progenitor cell therapy in peripheral artery disease. A critical appraisal. Thromb Haemost 2010; 103: 696-709.

29. Charwat S, Lang I, Dettke M, et al. Effect of intramyocardial delivery of autologous bone marrow mononuclear stem cells on the regional myocardial perfusion. NOGA-guided subanalysis of the MYSTAR prospective randomised study. Thromb Haemost 2010; 103: 564-571.

30. Murry CE, Keller G. Differentiation of embryonic stem cells to clinically relevant populations: lessons from embryonic development. Cell. 2008; 132: 661-680.

31. Dimos JT, Rodolfa KT, Niakan KK, et al. Induced pluripotent stem cells generated from patients with ALS can be differentiated into motor neurons. Science 2008; 321: 1218-1221.

32. Si-Tayeb K, Noto FK, Nagaoka M, et al. Highly efficient generation of human hepatocyte-like cells from induced pluripotent stem cells. Hepatology 2010; 51: 297-305.

33. Song Z, Cai J, Liu Y, et al. Efficient generation of hepatocyte-like cells from human induced pluripotent stem cells. Cell Res 2009; 19: 1233-1242.

34. Zhang D, Jiang W, Liu M, et al. Highly efficient differentiation of human ES cells and iPS cells into mature pancreatic insulin-producing cells. Cell Res 2009; 19 $429-438$.
35. Xu D, Alipio Z, Fink LM, et al. Phenotypic correction of murine hemophilia A using an iPS cell-based therapy. Proc Natl Acad Sci USA 2009; 106: 808-813.

36. Hanna J, Wernig M, Markoulaki S, et al. Treatment of sickle cell anemia mouse model with iPS cells generated from autologous skin. Science 2007; 318: 1920-1923.

37. Ye L, Chang JC, Lin C, et al. Induced pluripotent stem cells offer new approach to therapy in thalassemia and sickle cell anemia and option in prenatal diagnosis in genetic diseases. Proc Natl Acad Sci USA 2009; 106: 9826-9830.

38. Carr AJ, Vugler AA, Hikita ST, et al. Protective effects of human iPS-derived retinal pigment epithelium cell transplantation in the retinal dystrophic rat. PLoS One 2009; 4: e8152.

39. Nelson TJ, Martinez-Fernandez A, Yamada S, et al. Repair of acute myocardial infarction by human stemness factors induced pluripotent stem cells. Circulation 2009; 120: 408-416.

40. Zwi L, Caspi O, Arbel G, et al. Cardiomyocyte differentiation of human induced pluripotent stem cells. Circulation 2009; 120: 1513-1523.

41. Lian Q, Zhang Y, Zhang J, et al. Functional mesenchymal stem cells derived from human induced pluripotent stem cells attenuate limb ischemia in mice. Circulation 2010; 121: 1113-1123.

42. Hong H, Takahashi K, Ichisaka T, et al. Suppression of induced pluripotent stem cell generation by the p53-p21 pathway. Nature 2009; 460: 1132-1135.

43. Utikal J, Polo JM, Stadtfeld M, et al. Immortalization eliminates a roadblock during cellular reprogramming into iPS cell. Nature 2009; 460: 1145-1148.

44. Esteban MA, Wang T, Qin B, et al. Vitamin C enhances the generation of mouse and human induced pluripotent stem cells. Cell Stem Cell 2010; 6: 71-79.

45. Yamanaka S. A fresh look at iPS cells. Cell 2009; 137: 13-17.

46. Lee AS, Tang C, Cao F, et al. Effects of cell number on teratoma formation by human embryonic stem cells. Cell Cycle 2009; 8: 2608-2612. 